\title{
Desarrollo de Tablas de Evaluación y Factores de Ponderación del Riesgo Asociado a Tipos de Técnicas de Manipulación en Tareas con Manipulación Manual de Cargas Dinámico-Asimétricas
}

\author{
DEVELOPMENT OF EVALUATION TABLES AND WEIGHTING FACTORS OF RISK ASSOCIATED TO TYPES OF \\ HANDLING TECHNIQUES IN MANUAL HANDLING TASKS OF DYNAMIC-ASYMMETRIC LOADS
}

\author{
Eduardo Cerda Díaz', Álvaro Besoain Saldaña', Giovanni Olivares Péndola', Rodrigo Pinto Retamal², Carolina Rodriguez Herrera', \\ Jorge Rodriguez Tobar ${ }^{3}$, Claudio Rozbaczylo Fuster ${ }^{4}$ \\ 1. Investigador. Laboratorio de Ergonomía. Departamento de Kinesiología. Facultad de Medicina. Universidad de Chile. Santiago, Chile. \\ 2. Investigador Científico Asociación Chilena de Seguridad (ACHS). Santiago, Chile. \\ 3. Jefe División Bioestadistica. Escuela de Salud Pública. Universidad de Chile. Santiago, Chile. \\ 4. Laboratorio de Análisis de Movimiento Humano. Departamento de Kinesiología. Facultad de Medicina. Universidad de Chile. Santiago, Chile.
}

\begin{abstract}
RESUMEN
Objetivo: Describir factor de ponderación de riesgo del factor técnica de manipulación combinada (5 técnicas), analizando sus diferencias en condición dinámico-asimétrica, considerando la carga biomecánica en los segmentos extremidades superiores y columna. Método: Estudio cuasiexperimental, analítico y transversal, muestra por conveniencia de 100 sujetos (hombres y mujeres) en edad laboral, de la comunidad universitaria de la Universidad de Chile, evaluando cinco técnicas de manipulación de carga, utilizando sistema optoelectrónico, 3D Static Strength Prediction Program, electromiografía de superficie y percepción de esfuerzo. Resultados: En extremidades superiores, se describen diferencias significativas entre técnicas en todas las variables de análisis biomecánico: el patrón de torque, activación muscular y percepción de esfuerzo es mayor en la técnica simétrica sobre hombros y asimétrica que en las otras técnicas. A nivel de columna, la actividad electromiográfica a nivel de erectores espinales entre técnicas es estadísticamente significativa. Conclusión: Las técnicas con mayor carga biomecánica son las técnicas "Simétrica sobre Hombro" y "Asimétrica". La técnica "Simétrica a nivel de caderas" genera una carga intermedia, las técnicas "Simétrica a nivel de tronco" y de "Una mano" tienden a ser similares y las menos exigentes.
\end{abstract}

(Cerda E, Besoain A, Olivares G, Pinto R, Rodriguez C, Rodriguez J, Rozbaczylo C, 2015. Desarrollo de Tablas de Evaluación y Factores de Ponderación del Riesgo Asociado a Tipos de Técnicas de Manipulación en Tareas con Manipulación Manual de Cargas Dinámico-Asimétricas. Cienc Trab. May-Ago; 17 [53]: 115-121).

Palabras claves: INGENIERÍA HUMANA, ESFUERZO FÍSICO, CARGA DE TRABAJO, ANÁLISIS Y DESEMPEÑO DE TAREAS.

\section{ABSTRACT}

To describe risk weighting factor of combined manipulation technique factor ( 5 Technical), analyzing their differences in dynamicasymmetrical condition, considering the biomechanical load on the upper limbs and spine segments.

Method: Quasi-experimental, analytical and cross-sectional study, convenience sample of 100 subjects (male and female) in working age, from University of Chile community, assessing five load-handling techniques using optoelectronic system, 3D Static Strength Prediction Program, surface electromyography and perceived exertion.

Results: in upper extremities, significant differences in all variables techniques of biomechanical analysis are described: the pattern of torque, muscle activation and perceived exertion is higher in the symmetric on shoulders and asymmetric technique than the other techniques. In a column level, the electromyographic activity in a spinal erector level between techniques is statistically significant. Conclusion: The techniques with more biomechanical loading are "Symmetric on Shoulder" and "asymmetric" techniques. The "symmetrical hips level" technique generates an intermediate charge, techniques "trunk level Symmetric" and "One hand" tend to be similar and less demanding.

Keywords: HUMAN ENGINEERING, PHYSICAL EFFORT, WORKLOAD, TASK PERFORMANCE AND ANALYSIS.
Correspondencia / Correspondence:

Eduardo Cerda Díaz

Av. Independencia 1027

Santiago, Chile

Laboratorio de Ergonomía. Departamento de Kinesiología

Facultad de Medicina. Universidad de Chile

e-mail: encerda@med.uchile.cl.

Tel. - Fax: $9786513 / 9786520$

Recibido: 21 de Febrero de 2015 / Aceptado: 17 de Abril de 2015

\section{INTRODUCCIÓN}

En el marco del estudio ergonómico de tareas con Manipulación Manual de Carga Dinámico-Asimétricas (MMCDA), la cual se define como la ejecución de manipulación manual de carga con levantamiento, transporte y levantamiento en forma continua y en perímetros mayores a dos metros. ${ }^{1}$ Existen factores que se deben tomar en consideración cuando se pretende evaluar el riesgo de dicha manipulación, entre los cuales se considera relevante el factor de técnica de manipulación, basando su análisis en el comportamiento biomecá- 
nico del posicionamiento de los brazos en el desarrollo de una actividad específica, en relación al tronco y sus efectos en relación a la carga biomecánica, específicamente a nivel de segmento columna y miembros superiores.

En este contexto, la consecuencia de la postura de brazos en las diferentes técnicas está dada por el resultado de la interacción de la persona con el entorno. En el caso de las técnicas de manipulación existen distintas combinaciones de técnicas que implican una mayor o menor carga biomecánica (Carga Física) y a su vez riesgo para la salud musculoesquelética de las personas, basado este concepto esencialmente en el nivel de carga biomecánica exigido en los segmentos expuestos. ${ }^{2,3}$

En entornos complejos, tales como el sector de la construcción y otros sectores productivos de análisis complejo del riesgo en tareas con manipulación manual de carga, el número de los diferentes materiales manipulados durante la jornada de trabajo es elevado, por lo que el análisis de cada uno de los materiales es poco viable. Por lo anterior, la evaluación del riesgo en base al análisis de los mismos se torna un proceso largo y poco preciso, obteniendo evaluaciones sin una confiabilidad adecuada, debido a la discordancia entre la realidad de los procesos productivos y el constructo de los instrumentos de evaluación actuales. ${ }^{4}$

Ante estos factores, se plantea que es necesario avanzar en estrategias de evaluación alternativas. ${ }^{5,6}$ Una propuesta ante este problema es el cambio de estrategia de evaluación, expuesto en el Congreso Mundial de Ergonomía de Maastrich por los autores del Método EC 2 y en el circuito científico local. ${ }^{1,7}$ En este modelo de evaluación, se define una estrategia enfocada en el análisis de la técnica de manipulación y no en los materiales que se puedan manipular. Es así que en el proceso de evaluación se pueden distinguir diferentes combinaciones de técnicas de manipulación, a fin y efecto de considerarlas finalmente, junto a todas las otras variables observadas, como postura de manipulación, tipo de agarre o dificultad de manipulación en base a condiciones del entorno o elementos utilizados, entre otros. Se ha observado que en el proceso de evaluación, este conjunto de variables están presentes en tareas con manipulación manual dinámico-asimétricas presentando asociaciones significativas. ${ }^{8}$

En este sentido, dentro de todas las variables que actúan en la evaluación del riesgo de trastornos musculoesqueléticos asociados a las Manipulaciones Manuales de Carga propuestas en el método EC2 (ErgocargaConstrucción-EC2), basado en el método NIOSH, se agregan 4 factores de riesgos, siendo estos: Técnica de manipulación combinada, Postura de manipulación combinada, Factor de esfuerzo percibido y Factor de dificultad de manipulación.

Se propone posteriormente una nueva propuesta de Ecuación de Cálculo de Límite de Peso recomendado del Método EC2, donde permanecen solamente los factores que constituyen asimetría. Expresando la ecuación de la siguiente forma y tal como se muestra a continuación. ${ }^{9}$

El límite de peso recomendado se obtiene mediante la aplicación de una ecuación multiplicativa lineal que se describe a continuación:

Donde:

$$
\mathrm{LPR}=\mathrm{CC} \times \mathrm{FF} \times \mathrm{FTC} \times \mathrm{FPC} \times \mathrm{FAC} \times \mathrm{FEP} \times \mathrm{FDM}
$$

CC: Constante de carga

FF: Factor de frecuencia

FTC: Factor de técnica

FPC: Factor de postura combinada

FAC: Factor de agarre combinado

FEP: Factor de esfuerzo percibido

FDM: Factor de dificultad de manipulación
De esta manera, en esta investigación se profundiza en el análisis asociado al factor de Técnicas de manipulación combinada, el cual considera la asimetría de la manipulación manual durante la tarea y, con ello, la consecuente carga biomecánica en la misma, principalmente en los segmentos columna y miembros superiores. Este factor analiza por separado cada una de las técnicas de manipulación propuestas en el método EC2, que en base a la bibliografia son las más utilizadas en ambientes complejos de trabajo. ${ }^{7}$ La comprensión del fenómeno ocurrido en cada una de estas técnicas permitirá proyectar el análisis de técnicas combinadas planteadas en el método EC2. En etapas posteriores, este enfoque permitirá el análisis del factor Técnica de manipulación combinada e integrarlo junto a los otros factores planteados en la ecuación de método EC2.

En este contexto, el objetivo general de esta investigación es determinar factores de ponderación del riesgo para el factor Técnica de manipulación combinada (5 técnicas) en tareas con MMCDA, a través de la determinación del nivel de carga biomecánica en técnicas de manipulación con una mano, con ambas manos simétricas a nivel de tronco, con ambas manos simétricas a nivel de cadera, con ambas manos asimétricas a diferentes alturas, y con ambas manos simétricas por sobre nivel de hombro por medio de mediciones tanto en segmento miembro superior y columna, utilizando cinética, electromiografía de superficie y percepción de esfuerzo en laboratorio, en población en edad laboral de la comunidad universitaria de la Universidad de Chile.

A su vez, como proyección en base a los resultados, se propone el desarrollo de un instrumento de campo mediante tablas de evaluación orientadas a conocer el riesgo de diferentes técnicas de manipulación manual de carga dinámico-asimétrica, estableciendo una aproximación al riesgo inicial según tipo de técnicas de manipulación en condiciones de asimetría y estableciendo las bases para la priorización de evaluaciones más específicas en el desarrollo de este tipo de tareas.

\section{MATERIAL Y MÉTODOS}

\section{Muestra}

Se realiza un muestreo no probabilístico, mediante la selección por conveniencia de 100 personas (hombres y mujeres) en edad laboral y miembros de la comunidad universitaria de la Universidad de Chile, que acepten participar en el estudio. Se realiza una asignación homogénea según técnica de estudio por medio de un proceso aleatorio en bloque, lo cual permite distribuir 20 sujetos a cada técnica.

\section{Criterios de inclusión}

- Hombres y mujeres en edad laboral (18 a 65 años) que acepten voluntariamente participar en el estudio.

- Miembros de la comunidad de la Universidad de Chile. En el caso de que los sujetos de investigación sean estudiantes, y reconociendo una potencial situación de vulnerabilidad, se contemplarán medidas adicionales para proteger la confidencialidad de sus datos y para promover su autonomía, evitando cualquier situación que pudiese entenderse como presión para aceptar participar en el estudio.

\section{Criterios de exclusión}

- Sujetos en edad laboral con patología de columna diagnosticada y con diagnóstico de patología de extremidad superior diagnosticada en los últimos 12 meses. 
- Sujetos que presenten enfermedades sistémicas y/o autoinmunes (Artritis Reumatoide, Fibromialgia, Neuropatías, etc.).

- Se excluyen del estudio mujeres embarazadas.

\section{Diseño y tipo de investigación}

El presente estudio se define como de tipo analítico. Su diseño de investigación es de tipo transversal, cuasiexperimental.

\section{Protocolo de Medición}

El presente estudio considera el análisis y evaluación de factores biomecánicos en técnicas de manipulación en tareas con MMCDA realizadas en el Laboratorio de Ergonomía, en conjunto con el Laboratorio de Análisis del Movimiento Humano de la Universidad de Chile, a través de la aplicación de sistemas integrados para el análisis cinemático tridimensional, cinética, electromiografía de superficie y percepción de esfuerzo a los sujetos que acepten participar de este.

El protocolo de estudio se estructura en base a consideraciones generales de caracterización de cada sujeto, como la medición de datos antropométricos de peso $(\mathrm{kg})$ y estatura $(\mathrm{m})$ de sujeto descalzo y lateralidad, y otras de carácter ético para asegurar confidencialidad, seguridad, confort y autonomía de los participantes, a través de la entrega de un consentimiento informado, además de la asignación de un número de ficha independiente para cada uno. Los materiales requeridos para las mediciones consistieron en sistema BTS Smart, Programa 3DSSPP, equipo de electromiografía BTS, equipo BTS (3D) calibrado, 6 electrodos de superficie y 12 pegatinas por persona, además de 24 marcadores reflectantes, gel, alcohol, algodón, tela adhesiva, camilla y, por último, el objeto a manipular. Con el fin de asegurar un protocolo seguro, basado en los lineamientos científicos en el área y en relación a las características del material a ser manipulado (barra Z), se define el peso de la misma con una carga final equivalente a $7 \mathrm{~kg}$ de peso, que corresponde a 69,6465 $\mathrm{N}$ de fuerza, diseñando de esta forma un protocolo, considerando una condición sin riesgo, sustentado en base a referencias técnicas. ${ }^{10,11}$

En relación al sendero de marcha, área en donde los sujetos son evaluados al realizar las diferentes técnicas del estudio, en esta zona están dispuestas las seis cámaras infrarrojas que captan los movimientos realizados, junto a otra cámara de video digital dispuesta por lateral. Las dimensiones del sendero son de 4,8 $\mathrm{m}$ de largo por 1,4 m de ancho. Los límites para el estudio son el punto situado a $2.1 \mathrm{~m}$ desde el inicio denominado "área de asimetría", hasta los 3,1 m que corresponde al área de depósito de la carga.

Para la preparación de cada sujeto, se definen 3 procedimientos: ubicación de electrodos para medición de electromiografía de superficie, según lo dictado por el proyecto SENIAM (Electromiografía de Superficie para la Evaluación no Invasiva de los Músculos) para los siguientes grupos musculares: trapecio superior, trapecio inferior y erectores espinales, todos en forma bilateral; localización de marcadores reflectantes según sistema optoelectrónico tridimensional (BTS Smart), que se utilizan para el análisis cinemático articular, que son situados en referencias anatómicas específicas (ver Tabla 1) y, por último, un procedimiento para definición de exploración de percepción de esfuerzo en ejecución de técnicas según escala CR-10 EESS ${ }^{12}$, la cual se presenta a cada sujeto una vez concluida la ejecución de la técnica asignada, y respondiendo según su nivel de percepción de esfuerzo en miembro superior. El criterio para definir la existencia del uso de fuerza considera el nivel 3 de la tabla (moderado), lo cual significa una percepción positiva por parte del evaluado para el uso de fuerza en la acción técnica; cualquier nivel igual o superior a este se considera como presencia de fuerza para el protocolo.
Tabla 1.

Zonas de distribución corporal de puntos de referencia para marcadores reflectantes.

\begin{tabular}{cc}
\hline & Marcadores de referencia \\
\hline Cabeza / EESS & Pelvis / EEIl \\
Oreja Izquierda & EIAS Derecha \\
Oreja derecha & EIAS Izquierda \\
Centro superior de la cabeza & L5S1 \\
Proceso espinoso C7 & Trocánter mayor derecho \\
Escotadura yugular (Manubrio esternal) & Trocánter mayor izquierdo \\
Articulación A-C Derecha & Cóndilo lateral rodilla derecha \\
Articulación A-C Izquierda & Cóndilo lateral rodilla izquierda \\
Olécranon derecho & Maleólo lateral derecho \\
Olécranon izquierdo & Maleólo lateral izquierdo \\
Ap. estiloides Radio derecha & $3^{\circ}$ MTT derecho \\
Ap. estiloides Radio izquierda & $3^{\circ}$ MTT izquierdo \\
Ap. Estiloides ulnar derecha & \\
Ap. Estiloides ulna izquierdar & \\
\hline
\end{tabular}

Algunas consideraciones técnicas presentes en el estudio son que, previo a los procedimientos de ejecución de prueba de laboratorio según técnica de manipulación y evaluación de máxima contracción voluntaria (MCV) por sujeto, se realiza un chequeo del modelo biomecánico diseñado en el sistema optoelectrónico, compuesto de 24 puntos de referencia según Tabla 1, en los cuales se instalan los marcadores reflectantes, cuyo movimiento se captura por el sistema para obtener la orientación espacial de los segmentos corporales y, de esta manera, estimar la variación angular (cinemática) articular. La unión de estos 24 puntos de referencia dan origen a 14 segmentos de referencia reconstruidos según el modelo creado, donde se puede distinguir: cabeza, cintura escapular, brazo izquierdo, brazo derecho, antebrazo izquierdo, antebrazo derecho, tronco, pelvis, muslo derecho, muslo izquierdo, crura (pierna) derecha, crura (pierna) izquierda, tobillo derecho y tobillo izquierdo. Además, también se chequea la codificación de electrodos de EMG según protocolo, en base a las zonas específicas para la ubicación de los electrodos de electromiografía de superficie recomendadas por el SENIAM, en relación a la musculatura a analizar. Para el registro electromiográfico del total de los sujetos participantes del estudio se utiliza un total de 2000 electrodos de plata/cloruro de plata $(\mathrm{Ag} / \mathrm{AgCl}$ marca Kendall, modelo Medi-trace 100).

Para las diferentes técnicas a estudiar, los análisis de éstas se llevan a cabo bajo el supuesto de que los movimientos de elevación son fluidos, y que la distribución de la carga de ambas manos sea simétrica, y la fuerza se considera que es directamente vertical y hacia abajo. En relación a la postura analizada, se identifica el momento (en segundos) durante el desplazamiento del sujeto por el sendero de marcha, cuando este supere los 2,1 m desde el punto de partida, tomando como referencia la activación de la plataforma de fuerza situada en el punto medio del sendero cercano a los 2,1 m, definido como punto de asimetría. Es en este punto donde se vinculan las distintas variables cuantificadas, es decir, la obtención de datos de cinemática articular y EMG.

Posteriormente, las medidas angulares de los distintos segmentos son vertidas en el programa 3DSSPP para así estimar la carga biomecánica para cada una de las técnicas dinámico-asimétricas, así como en las diferentes partes del cuerpo en forma individuales, a través del cálculo de torques o momentos de fuerza para hombro y zona L5/S1.13 
Figura 1.

Descripción de técnicas a ejecutar en laboratorio según técnica de manipulación.

\begin{tabular}{|c|c|c|}
\hline Técnica & Descripción gráfica & Descripción \\
\hline Técnica a nivel de tronco & & $\begin{array}{l}\text { Sujeción de material } \\
\text { con flexión de } 90 \text { grados de } \\
\text { codo con brazo paralelo } \\
\text { a tronco. }\end{array}$ \\
\hline $\begin{array}{l}\text { Técnica Simétrica sobre } \\
\text { nivel de hombro }\end{array}$ & & $\begin{array}{l}\text { Sujeción de material con } \\
\text { flexión sobre el nivel } \\
\text { del hombro. }\end{array}$ \\
\hline Técnica Asimétrica & & $\begin{array}{l}\text { Sujeción de material con } \\
\text { una mano próxima a la } \\
\text { línes de hombro a la altura } \\
\text { de ojo y otra mano a la } \\
\text { altura de las caderas. }\end{array}$ \\
\hline Técnica a nlvel de caderas & & $\begin{array}{l}\text { Sujeción de material con } \\
\text { brazos estirados } \\
\text { levemente separados del } \\
\text { cuerpo bajo el nivel } \\
\text { de las caderas. }\end{array}$ \\
\hline Técnica con una mano & & $\begin{array}{l}\text { Sujeción de material con } \\
\text { una mano con brazo } \\
\text { estirado por lateral } \\
\text { separado del cuerpo. }\end{array}$ \\
\hline
\end{tabular}

Cada sujeto se evalúa realizando las pruebas mirando al frente, manteniendo el tronco en posición vertical y simétrica, estandarizando cada técnica, según Figura 1.

Cabe destacar que las acciones a ejecutar durante la prueba en laboratorio fueron estipuladas dentro de una "hoja de campo de laboratorio", en forma específica para cada una de las 6 etapas descritas que se resumen en:

- Preparación de prueba

- Ubicación de electrodos

- Ubicación de marcadores reflectantes

- Revisión de calibración y funcionamiento de sistema

- Prueba de laboratorio y exploración de percepción de esfuerzo

- Evaluación MCV

Teniendo todo este proceso un tiempo total estimado por sujeto de 55 minutos.

Cabe señalar que el registro electromiográfico se realiza de manera sincronizada con la captura de movimiento tridimensional. Para ello se utiliza equipo de EMG modelo FreeEMG de 6 canales (BTS Bioengineering, Italia), con una frecuencia de registro de $1000 \mathrm{~Hz}$. Se disponen electrodos de superficie $(\mathrm{Ag} / \mathrm{AgCl})$ de acuerdo a las recomendaciones del SENIAM.

La actividad electromiográfica de los músculos seleccionados para este estudio (trapecio ascendente, trapecio descendente y erector espinal, todos bilateralmente) se mide de manera dinámica durante la ejecución de las 5 técnicas de manipulación. En cada ocasión se define una fase de ascenso del implemento, una fase de descenso del implemento y una fase intermedia, en la cual el sujeto sostiene el implemento mientras camina hacia la posición final, pasando por el punto de asimetría.

El procesamiento de las señales de EMG consiste en un filtro pasa alto de $7 \mathrm{~Hz}$ (Hamming) para eliminar los componentes de ruido eléctrico proveniente del movimiento de los propios electrodos, los cables o la piel del sujeto bajo los electrodos. A continuación, la señal electromiográfica se rectifica en onda completa y luego se obtiene su envolvente mediante la aplicación de un filtro RMS (Root Mean Square) con una ventana temporal móvil de $250 \mathrm{mseg}$, que crea una señal envolvente más sencilla de leer y procesar.

Una vez procesada esta señal dinámica, y para estimar el nivel de actividad electromiográfica utilizada en cada fase y por cada músculo, se compara ésta con la realizada por el sujeto en una contracción voluntaria máxima (CVM), basado en las pruebas funcionales musculares de Daniels/Worthingham's para cada grupo muscular (14), a las que se somete cada sujeto luego de haber realizado la ejecución de la técnica asignada $\mathrm{y}$, por tanto, se calcula para cada músculo la proporción que representa su actividad durante la tarea dinámica respecto a los valores obtenidos en la contracción voluntaria máxima (CVM).

Paralelamente, se realiza un vaciamiento de datos de la información obtenida de los resultados de la fuerza de compresión a nivel de L5/S1 en el plano sagital lumbar, utilizando la unidad de medida Newton. El sistema 3DSSPP utiliza un análisis de la fuerza de compresión del disco L5/S1, basado en un modelo predictivo usando las fuerzas de contracción de los músculos erectores de la columna y recto abdominal. En tanto que, para los Momentos de fuerza (Torque), expresados en unidad de medida Newton por metro (Nxm), producidos por la carga y el peso corporal de cada sujeto, el programa calcula estos utilizando un modelo biomecánico basado en un algoritmo de vinculación estática, que dependen de la antropometría, la postura y la carga de la mano del sujeto, que en este caso corresponde a $7 \mathrm{~kg}(69,6465 \mathrm{~N})$. Finalmente, con la información procesada según los datos ingresados, se obtienen los resultados correspondiente a fuerza de compresión a nivel L5/S1 y torques de hombro y L5/S1(13).

\section{ANÁLISIS DE DATOS}

Se utilizan estadísticos descriptivos de tendencia central y dispersión para resumir cada variable. Según la prueba de Shapiro y Wilks, la mayoría de los datos tendian a distribuir de manera no paramétrica, por lo que en la mayoría de los datos se utiliza la mediana y el rango intercuartílico.

Se realizan análisis globales del impacto de las variables Torque Hombro, torque L5/S1, Fuerza de Compresión L5/S1, Percepción Subjetiva del Esfuerzo y Actividad Eléctrica Muscular. En la construcción de los factores, sólo se consideran aquellas variables con diferencias significativas entre técnicas, tanto en miembro superior y columna. Desde la estadística inferencial, se utiliza la prueba de Mann-Whitney para la comparación entre parejas, mientras que para comparar entre más de dos categorías, se utiliza la prueba de Kruskall-Wallis, junto a la prueba de Bernard Rosner como estadístico no paramétrico post-hoc. Este análisis se desglosa en las variables asociadas a Extremidades Superiores (EESS) y Columna. Finalmente, y posterior a este procedimiento, se determinan las variables que se utilizan posteriormente en la construcción de ponderadores según técnica.

La construcción de los factores de ponderación se obtiene a través de modelo matemático específico, cuyo objetivo es obtener factores que sigan la línea de interpretación de la Ecuación de NIOSH, donde cada factor está comprendido entre los valores 0 y 1 . En ese contexto, un factor de ponderación representa una situación crítica 
mientras más próximo se encuentre al valor $0 \mathrm{y}$ una condición ideal mientras más próximo a 1.

El modelo matemático se basa en cálculo de medianas para cada variable de estudio por técnicas y posteriormente una comparación en forma individual y global de éstas. A su vez, el modelo matemático realiza integración en la construcción de los factores finales, los de los criterios del valor mínimo del factor multiplicativo horizontal y vertical de la Ecuación de NIOSH.

Se concluye el proceso promediando los factores de ponderación finales por variable y técnicas para Miembro Superior y Columna, obteniendo de esta forma un factor de ponderación global por cada una de las técnicas analizadas.

\section{RESULTADOS}

Un total de 100 personas fueron evaluadas, distribuidas de manera homogénea y equitativa en los 5 tipos de técnica (20 personas por técnica). La relación de la cantidad de hombres/mujeres fue de 1,08 (52/48). En la tabla a continuación se describen las características de la muestra. Se realiza la prueba de Shapiro y Wilks para identificar el tipo de distribución de las variables independientes o características de los datos, la cual arroja que estas variables distribuian normal. En general, la muestra obtenida consta de personas de la comunidad universitaria en edad laboral normopeso, sin diferencias estadísticamente significativas entre los grupos generados por cada técnica, por medio de la prueba de ANOVA. A continuación se entrega una tabla que resume estos valores.

Las variables específicas de análisis están divididas entre tres gran-

Tabla 2.

Característica de la muestra. Se describe la mediana. Prueba de ANOVA; alfa $=5 \%$.

$\begin{array}{lllllll} & \begin{array}{l}\text { Técnica a } \\ \text { nivel de } \\ \text { tronco }\end{array} & \begin{array}{l}\text { Técnica } \\ \text { Simétrica } \\ \text { sobre hombros }\end{array} & \begin{array}{l}\text { Técnica } \\ \text { Asimétrica }\end{array} & \begin{array}{l}\text { Técnica a } \\ \text { nivel de } \\ \text { caderas }\end{array} & \begin{array}{l}\text { Técnica } \\ \text { con una } \\ \text { mano }\end{array} & \begin{array}{l}\text { Diferencias } \\ \text { entre } \\ \text { técnicas* }\end{array} \\ \text { Edad (años) } & 23,50 & 23,00 & 24,00 & 23,00 & 23,50 & p=0,169 \\ \text { Talla }(\mathrm{m}) & 1,69 & 1,65 & 1,68 & 1,69 & 1,67 & \mathrm{p}=0,351 \\ \text { Peso }(\mathrm{Kg}) & 64,35 & 65,65 & 66,30 & 70,50 & 67,25 & \mathrm{p}=0,411 \\ \text { IMC } & 23,23 & 23,23 & 24,49 & 25,28 & 23,78 & \mathrm{p}=0,503\end{array}$

des ejes. Primero, variables asociadas a Cinética articular (Torque de hombro, Torque de columna y fuerza de compresión a nivel de L5/S1); Segundo, variables asociadas a actividad electromiográfica (Trapecio Superior, Trapecio Inferior y Erectores Espinales), y percepción subjetiva de esfuerzo a nivel de EESS. Como se describe en el método, las variables independientes presentes en este estudio son la técnica utilizada por cada grupo y el género de los sujetos. Por ello se realiza análisis globales del impacto de dichas variables, junto a un desglose entre-género y entre-técnica.

\section{Comparación entre técnicas}

A nivel de EESS, se identifican diferencias significativas en todas las variables entre técnicas. En general, el patrón de torque, activación muscular y percepción de esfuerzo es mayor en la técnica simétrica de hombros y asimétrica que en las otras técnicas; sin embargo, el músculo trapecio superior derecho presenta un valor muy cercano al límite establecido $(\mathrm{p}=0,022)$. La técnica con menos factores de riesgos ergonómicos para EESS en el puesto de trabajo, fue la técnica con una sola mano.
Realizando el análisis de comparación entre técnicas separando por género, se identifica que la actividad eléctrica muscular del trapecio superior izquierdo o derecho es igual, tanto en hombres como mujeres en la mayoría de las técnicas.

Además, no existen diferencias en la percepción de esfuerzo entre técnicas para los hombres $(\mathrm{p}=0,438)$, mientras que las mujeres perciben más esfuerzo en la técnica simétrica sobre hombros y asimétrica que realizando otras.

El torque a nivel de hombro presenta diferencias estadísticamente significativas entre técnicas, tanto para hombres $(p<0,001)$ como para mujeres ( $\mathrm{p}<0,001)$.

A nivel de columna, sólo la actividad electromiográfica a nivel de Erectores espinales entre técnicas fueron estadísticamente significativas, tanto los músculos derechos e izquierdos ( $p<0,001$ para ambos). Al realizar un análisis entre técnicas por cada género por separado, los resultados anteriormente mencionados se mantienen. En base a estos hallazgos, se posee como resultado las variables que deben ser incluidas en la generación de los factores y para las cuales se puede establecer una comparación entre técnicas; estas serian:

- A nivel de miembro superior: Torque de Hombro, Percepción de Esfuerzo, Actividad eléctrica muscular de trapecios (superiores e inferiores).

- A nivel de columna: Actividad eléctrica muscular de erectores espinales derechos e izquierdos.

Considerando los objetivos planteados en esta investigación, se prioriza la generación de factores de ponderación, tomando en consideración las diferencias entre técnicas, tal como se ha descrito en el texto. Los resultados obtenidos nos presentan un comportamiento variable en relación a las diferencias significativas entre género, lo que limita la generación de factores diferenciados por género.

\section{Factores de Ponderación}

Los factores finales por cada variable y por cada técnica se presentan en la siguiente tabla. Sólo fueron incluidas las variables con diferencias estadísticamente significativas entre las técnicas. Las diferencias entre las otras técnicas son más leves, pero se puede afirmar que el patrón nuevamente se confirma, posicionando a la técnica de una mano como la menos impactante a nivel de carga biomecánica.

\section{Tabla 3.}

Factores de ponderación para Miembro Superior. En esta tabla se entrega cada Factor Final por variable.

\begin{tabular}{|c|c|c|c|c|c|c|c|c|}
\hline & TH & CR10 & TSD & TSI & TID & TII & $\begin{array}{l}\text { Factor } \\
\text { Final } \\
\text { (d.e.) }\end{array}$ & $\begin{array}{l}\text { Intervalo } \\
\text { de } \\
\text { Confianza }\end{array}$ \\
\hline $\begin{array}{l}\text { Técnica a } \\
\text { nivel de } \\
\text { tronco }\end{array}$ & 0,592 & 0,680 & 0,633 & 0,6286 & 0,5916 & 0,6127 & $0,623(0,03)$ & $0,56-0,69$ \\
\hline $\begin{array}{l}\text { Técnica } \\
\text { Simétrica } \\
\text { sobre } \\
\text { hombros }\end{array}$ & 0,521 & 0,564 & 0,520 & 0,4862 & 0,4703 & 0,4583 & $0,503(0,04)$ & $0,43-0,58$ \\
\hline $\begin{array}{l}\text { Técnica } \\
\text { Asimétrica }\end{array}$ & 0,512 & 0,535 & 0,516 & 0,5376 & 0,4746 & 0,5144 & $0,515(0,02)$ & $0,47-0,56$ \\
\hline $\begin{array}{l}\text { Técnica a } \\
\text { nivel de } \\
\text { caderas }\end{array}$ & 0,610 & 0,607 & 0,668 & 0,6304 & 0,5215 & 0,5410 & $0,596(0,06)$ & $0,49-0,70$ \\
\hline $\begin{array}{l}\text { Técnica } \\
\text { con una } \\
\text { mano }\end{array}$ & 0,715 & 0,564 & 0,612 & 0,7426 & 0,8919 & 0,8225 & $0,725(0,12)$ & $0,48-0,97$ \\
\hline
\end{tabular}


Tabla 4.

Factores Ponderación para Columna. En esta tabla se entrega cada Factor Final por variable relevante.

\begin{tabular}{lcccc} 
Técnica & \multicolumn{2}{c}{ Columna } & Factor & IC \\
Técnica a nivel de tronco & 0,966 & 0,934 & $0,95(0,02)$ & $0,91-0,99$ \\
Técnica Simétrica sobre hombros & 0,835 & 0,820 & $0,83(0,01)$ & $0,81-0,85$ \\
Técnica Asimétrica & 0,837 & 0,822 & $0,83(0,01)$ & $0,81-0,85$ \\
Técnica a nivel de caderas & 0,919 & 0,960 & $0,94(0,03)$ & $0,88-0,99$ \\
Técnica con una mano & 0,966 & 0,964 & $0,97(0,01)$ & $0,96-0,97$
\end{tabular}

C5: Erectores Espinales Derechos; C6: Erectores Espinales Izquierdos. El factor Final se entrega junto a su desviación estándar entre paréntesis. De: Desviación Estándar.

\section{CONCLUSIÓN Y DISCUSIÓN}

En esta investigación, cuyo principal objetivo es la determinación de factores de ponderación del riesgo para el factor Técnica de manipulación combinada (5 técnicas), analizando sus diferencias en condición dinámico-asimétrica, considerando la carga biomecánica en los segmentos miembro superior y columna, se identificó que las técnicas que implican mayor carga biomecánica son la técnica "Simétrica sobre Hombro" y la técnica "Asimétrica". La técnica "Simétrica a nivel de caderas" tiende a generar una carga física intermedia, ya que la técnica "Simétrica a nivel de tronco" y la técnica de "Una mano" tienden a ser similares y las menos exigentes. Los factores desarrollados en esta investigación permiten definir técnicas que presentan más riesgo que otras, asumiendo que a mayor carga biomecánica habrá una mayor posibilidad de desarrollar un trastorno músculo-esquelético; esto basado en las teorías de la evolución de los trastornos músculo-esqueléticos, en las cuales las variables fuerza y calidad de movimiento son determinantes en la determinación del sobreesfuerzo. ${ }^{16}$

Se ha considerado el análisis integrado de los segmentos miembro superior y columna, así como también la integración de nuevas variables para obtener un resultado más preciso y específico. Para el análisis de la carga física (carga biomecánica), se consideran las variables en segmento miembro superior y columna: torque (análisis cinemático), fuerzas de compresión (Análisis cinético), Actividad eléctrica muscular (Análisis Fisiológico), Percepción del esfuerzo (Análisis Psicofísico). Es necesario destacar que existen diferencias entre técnicas, tanto en Miembro Superior como en Columna, ya que en investigaciones previas los estudios se han centrado principalmente en el estudio de columna, así como también en torque y fuerza de compresión de columna.

Por otro lado, la diferencia obtenida entre los datos obtenidos de columna y miembro superior es coherente con la metodología de este estudio, ya que las posturas utilizadas para realizar las tareas de manejo de manual de carga en laboratorio se realizan en forma controlada con la columna alineada a línea media en el plano sagital y frontal, centrando el foco de investigación en las técnicas de manipulación propiamente tal.

Dentro de las limitaciones de este trabajo, es que las variables dependientes analizadas presentan una dispersión, expresado en sus rangos intercuartílicos, desviaciones estándar e intervalo de confianza. No obstante, es necesario destacar que la técnica "Una mano" es la que entrega menos carga biomecánica, pero presenta mayor variabilidad, por lo que su uso como la técnica más segura debe ser tomado con precaución. En este contexto, se sugiere que en situaciones de evaluación en terreno se apliquen evaluaciones más específicas para dicho sistema de trabajo, para conocer el riesgo efectivo de una tarea, junto a incluir las características del sistema de trabajo, por medio de metodologías con un constructo orientado a estudiar el riesgo de tareas con manipulación manual de carga dinámico asimétrica, tales como el Método EC2.

Cabe considerar que la variable "peso a movilizar" ha sido controlada en esta investigación, asignando un peso similar para cada una de las técnicas empleadas durante la prueba de laboratorio, por lo que analizar el comportamiento de los factores de ponderación ante distintos pesos, estableciendo mayor dificultad y fatiga en la ejecución, es una proyección de futuros estudios y que permitan continuar profundizando sobre el conocimiento en esta materia.

Tal como se ha propuesto en esta investigación, el objetivo central es diferenciar qué ocurría con la carga física (carga biomecánica) según tipos de técnicas. Este objetivo se ha logrado con éxito. Por otra parte, será necesario determinar factores de ponderación asociados a las otras variables de relevancia en el estudio de tareas con manipulación manual de carga dinámico-asimétricas, siendo estas: a) Factor de agarre combinado; b) Factor de postura de manipulación combinada; y c) Factor esfuerzo percibido.

Con lo anterior se proyecta generar para cada una de las variables del método EC2 una hoja de campo para evaluación en sectores complejos, proyectando la estructuración de un set de hojas de campo integradas que permitan la evaluación parcializada del riesgo según variable $y$, a su vez, en caso que se requiera converger todas en una evaluación más específica. 


\section{REFERENCIAS}

1. International Association of Ergonomics. World Congress of IAE. Ergonomics in the Construction Sector: The EC2 Method. Maastrich, Netherlands: International Association of Ergonomics; 2006.

2. Monnington SC, Pinder ADJ, Quarrie C. Development of an inspection tool for manual handling risk assessment [on line]. Sheffield, UK: Health and Safety Laboratory; 2003 [cited ene 2015]. Available on: http://www.hse.gov. uk/research/hsl_pdf/2002/hsl02-30.pdf

3. Takala EP, Pehkonen I, Forsman M, Hansson GA, Mathiassen SE, Neumann WP, Sjøgaard $G$ et al. Systematic Evaluation of Observational Methods Assessing Biomechanical Exposures at Work. Scand J Work Environ Health. 2010;36(1):3-24.

4. Dempsey P. Mathiassen S. On the Evolution of Task-Based Analysis of Manual Materials Handling, and its Applicability in Contemporary Ergonomics. Appl Ergon. 2006. 37(1):33-43.

5. Dempsey P. Utilizing Criteria for Assessing Multiple-Task Manual Materials Handling Jobs. Int J Ind Ergon. 1999;24(4):405-416.

6. Buchholz B, Paquet V. PATH: A Work Sampling-Based Approach to Ergonomic Job Analysis for Construction and Other Non-Repetitive Work. Appl Ergon.1996; 27(3):177-187.

7. Cerda $E$, Hernández A, Mondelo P, Álvarez E, Rodríguez C. La Ergonomía en el Sector Construcción: Método EC2. Cienc Trab. 2010;34:188-192.

8. Cerda E. Modelo Conceptual de Proceso de Evaluación de Factores Ergonómicos en Tareas con Manipulación Manual de Carga Dinámico
Asimétrica en el Sector de la Construcción [Tesis Doctorado] [on line]. Catalunya: Universitat Politecnica de Catalunya; 2013[citado ene 2015]. Disponible en: http://www.tesisenxarxa.net

9. Cerda E, Rodríguez $C$, Olivares $G$, Besoain A. Revisión de proceso de evaluación y fórmula de cálculo de límite de peso recomendado en Método EC2 para la Evaluación en Tareas con Manipulación Manual de Carga Dinámico Asimétrica. ORP Journal. 2014;1(1): 19-36.

10. International Organization for Standardization. ISO 11228-1:2010. Manual Handling. In Lifting and carrying. Geneva: ISO; 2010.

11. Instituto Nacional de Seguridad e Higiene del Trabajo (INSHT). Guía técnica para la evaluación y prevención de los riesgos relativos a la Manipulación Manual de Cargas. Madrid: Ministerio de Empleo y Seguridad Social; 2003.

12. Borg G. Borg's perceived exertion and pain scales. Champaign, IL: Human Kinetics; 1998.

13. University of Michigan. 3D-SSPP User's manual. 6.0.6 ed. Michigan, USA: University of Michigan; 2014.

14. Belen H, Dale A. Marybeth B. Daniels and Worthingham's Muscle Testing: Techniques of Manual Examination and Performance Testing. 9a ed. Madrid: Elsevier; 2014.

15 Waters T, Putz-Anderson V. Revised NIOSH Equation for the Design and Evaluation of Manual Lifting Tasks. Ergonomics. 1994;36(7):749-776.

16. Kumar S. Biomechanics in Ergonomics. London, MA: Taylor \& Francis; 1999. 